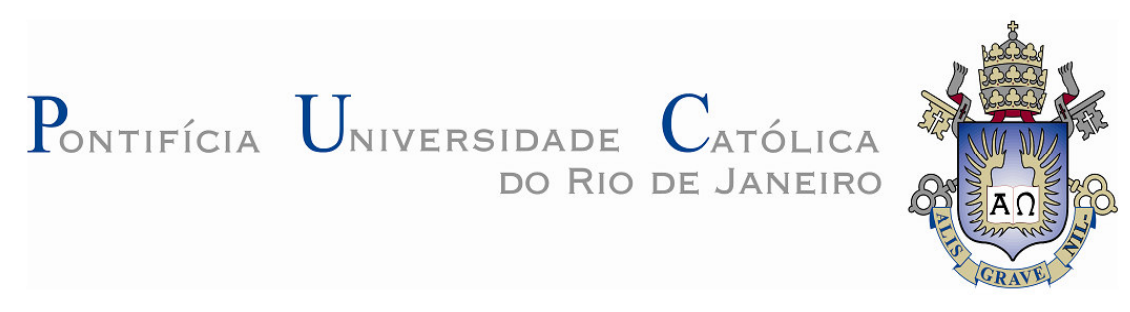

Miguel Angel Parodi Palacios

\title{
Comportamento de uma Areia Reforçada com Fibras de Polipropileno Submetida a Ensaios Triaxiais de Extensão
}

Dissertação apresentada ao Programa de PósGraduação em Engenharia Civil da como requisito parcial para obtenção do título de Mestre em Engenharia Civil.

Orientadora: Michéle Dal Toé Casagrande Co-orientador: Nilo Cesar Consoli 


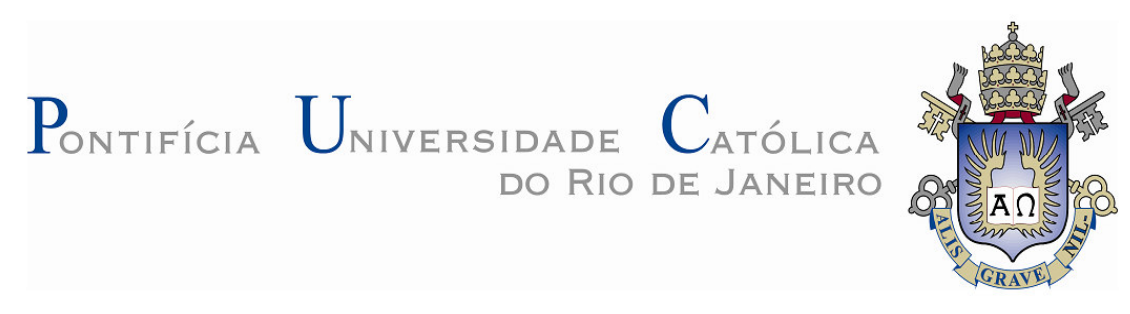

Miguel Angel Parodi Palacios

\title{
Comportamento de uma Areia Reforçada com Fibras de Polipropileno Submetida a ensaios Triaxiais de Extensão
}

\begin{abstract}
Dissertação apresentada como requisito parcial para obtenção do grau de Mestre pelo Programa de Pós-Graduação em Engenharia Civil da PUCRio. Aprovada pela Comissão Examinadora abaixo assinada.
\end{abstract}

\author{
Profa. Michéle Dal Toé Casagrande \\ Orientadora \\ Departamento de Engenharia Civil - PUC-Rio \\ Prof. Nilo Cesar Consoli \\ Co-orientador \\ Universidade Federal do Rio Grande do Sul
}

Prof. Silvrano Adonias Dantas Neto Universidade Federal do Ceará

Prof. Tácio Mauro Pereira de Campos Departamento de Engenharia Civil - PUC-Rio

Prof. José Eugenio Leal Coordenador Setorial do Centro

Técnico Científico - PUC-Rio

Rio de Janeiro, 03 de Maio de 2012. 
Todos os direitos reservados. É proibida a reprodução total ou parcial do trabalho sem autorização da universidade, do autor e da orientadora.

\section{Miguel Angel Parodi Palacios}

Graduou-se em Engenharia Civil pela Universidade Ricardo Palma (Lima-Peru) em 2007. Trabalhou em projetos e obras no Peru no período 2006-2009. Ingressou no mestrado na Pontifícia Universidade Católica do Rio de Janeiro em 2010, desenvolvendo Dissertação na linha de pesquisa de Geotecnia Experimental aplicada a solos reforçados.

Ficha Catalográfica

Parodi Palacios, Miguel Angel

Comportamento de uma areia reforçada com fibras de polipropileno submetida a ensaios triaxiais de extensão / Miguel Angel Parodi Palacios; orientadora: Michéle Dal Toé Casagrande ; co-orientador: Nilo Cesar Consoli. - 2012.

101 f. : il. (color.) ; $30 \mathrm{~cm}$

Dissertação (Mestrado)-Pontifícia Universidade Católica do Rio de Janeiro, Departamento de Engenharia Civil, 2012.

Inclui bibliografia

1. Engenharia civil - Teses. 2. Mistura solo-fibra. 3. Solo reforçado. 4. Ensaios triaxiais de extensão. 5. Resistência à tração. 6. Parâmetros de resistência. 7 . Fibras de polipropileno. I. Casagrande, Michéle Dal Toé. II. Consoli, Nilo Cesar. III. Pontifícia Universidade Católica do Rio de Janeiro. Departamento de Engenharia Civil. IV. Título.

CDD: 624 
Dedico esta Dissertação aos meus pais

Carlos Hernan e Ana Lucia, minha imensa gratidão e eterno amor por tudo que sempre fizeram por min; e aos meus irmãos Luis e Jose pelo companheirismo, apoio e força; a todos os familiares pelo carinho e incentivo. Tudo o que sou devo a vocês. 


\section{Agradecimentos}

Expresso a minha gratidão sincera a minha orientadora Michéle Dal Toé Casagrande pelos ensinamentos e as orientações dela recebidos desde 0 inicio do meu estudo e da minha pesquisa no mestrado até a conclusão desta dissertação e principalmente por sua amizade durante estes anos de convívio.

Ao professor Nilo Cesar Consoli pela disponibilidade de nos enviar a areia empregada na presente dissertação e pelas sugestões e recomendações nas diferentes etapas da pesquisa.

Ao professor Tácio Mauro Pereira de Campos pelas sugestões e orientações quanto à montagem do equipamento e a metodologia dos ensaios realizados no presente trabalho.

À Taíse Carvalho e Monica Moncada pela ajuda, auxílio e disposição fornecida sempre quando precisei no laboratório de geotecnia e meio ambiente da PUC-Rio.

Ao corpo docente do departamento de Engenharia Civil da PUC-Rio pelo ensino ministrado nas diferentes matérias as quais me serviram muito no aperfeiçoamento profissional. Obrigado professores, guardo muito respeito e admiração por cada um de vocês.

Ao professor Marco Antonio Hernandez Aguilar da Universidade Ricardo Palma (Lima-Peru), por ter acreditado em mim sempre, pelos primeiros ensinamentos da mecânica dos solos e por ter contribuído na minha formação em Geotecnia.

Á Jackeline Castañeda, pela sua ajuda, orientação e amizade 
desinteressada desde minha chegada ao Rio de Janeiro até o final do mestrado.

A todos os amigos e colegas pelos momentos agradáveis de convivência dentro e fora da PUC-Rio, obrigado por fazer destes dois anos uma das maiores experiências da minha vida.

À Pontifícia Universidade Católica do Rio de Janeiro e ao Programa de Pós-Graduação em Engenharia Civil, pela oportunidade de estudar nesta prestigiosa instituição.

À Química Suiza Peru. na pessoa do Eng. Milan Pejnovic, pelo fornecimento das fibras de polipropileno utilizadas nos ensaios realizados no presente estudo.

À CAPES, pelo apoio financeiro prestado para a concretização deste trabalho. 


\section{Resumo}

Palacios, Miguel Angel Parodi; Casagrande, Michéle Dal Toé; Consoli, Nilo Cesar. Comportamento de uma Areia Reforçada com Fibras de Polipropileno Submetida a Ensaios Triaxiais de Extensão. Rio de Janeiro, 2012. 101 p. Dissertação de Mestrado. Departamento de Engenharia Civil, Pontifícia Universidade Católica do Rio de Janeiro.

O presente trabalho permite avaliar o comportamento de um solo reforçado e não reforçado com fibras de polipropileno (teor de $0.5 \%$ em relação ao peso seco do solo) submetido a ensaios triaxiais de extensão, bem como a influência da adição de fibras na resistência e nos mecanismos de ruptura do solo reforçado. Para tanto, desenvolveu-se um programa experimental compreendendo a etapa final de um equipamento triaxial do tipo Bishop e Wesley de grandes dimensões e a execução de ensaios triaxiais drenados de extensão. Os ensaios triaxiais realizados permitiram a determinação dos parâmetros de resistência do solo e da mistura solo-fibra. Os resultados obtidos propiciaram a um melhor entendimento das alterações geradas pela inclusão aleatória de fibras de polipropileno à matriz da areia estudada. $\mathrm{O}$ desempenho das fibras no interior da massa de solo indica que estas sofreram deformações plásticas de tração, alongando-se, porém este alongamento é atribuído à fase de adensamento e não à fase de cisalhamento dos ensaios triaxiais de extensão realizados, já que foi possível observar que a adição das fibras não incrementa a resistência à tração do solo, quando este estiver submetido a esforços de extensão.

\section{Palavras-chave}

Mistura solo - fibra; solo reforçado; ensaios triaxiais de extensão; resistência à tração; parâmetros de resistência; fibras de polipropileno. 


\section{Abstract}

Palacios, Miguel Angel Parodi; Casagrande, Michéle Dal Toé (Advisor); Consoli, Nilo Cesar (Co-advisor). Behavior of a Polypropylene reinforced sand under Triaxial Extension Tests. Rio de Janeiro, 2012. 101 p. MSc. Dissertation - Civil Engineering Department, Pontifical Catholic University of Rio de Janeiro.

The present study allows evaluating the behavior of a reinforced and unreinforced soil with fibers of polypropylene $(0.5 \%$ content of dry weight of soil) subjected to triaxial extension tests, as the influence of adding fibers in the resistance and the rupture mechanisms of reinforced soil. Therefore, it was developed an experimental program comprising the development of a Bishop and Wesley's triaxial-type equipment which has a larger size than conventional triaxial equipment and an execution of drained triaxial extension tests. The present study submits triaxial tests results aiming the determination of strength parameters of soil and soil - fiber mixture. The obtained results led a better understanding of the alterations generated by the random inclusion of polypropylene fibers to the pattern sand studied. The accomplishment of fiber performance within soil mass indicates that they undergo tensile plastic deformation, elongating, but this elongating is attributed to shear stage in the triaxial extension tests realized, as was conferred that the addition of fiber does not increase the tensile strength of soil.

\section{Keywords}

Soil-fiber mixture; reinforced soil; triaxial extension tests; tensile strenght; strenght parameters, polypropylene fibers. 


\section{Sumário}

1 Introdução 17

$\begin{array}{ll}\text { 1.1 Relevância e justificativa da pesquisa } & 17\end{array}$

$\begin{array}{ll}1.2 \text { Objetivos } & 18\end{array}$

$\begin{array}{ll}1.3 \text { Organização da Dissertação } & 19\end{array}$

2 Revisão Bibliográfica 20

2.1 Considerações Iniciais $\quad 20$

2.2 Solos Reforçados $\quad 20$

2.2.1 Histórico, Melhoria, Estabilização e Reforço de Solos 20

2.2.2 Materiais Compósitos Reforçados com Fibras 23

2.2.3 Tipos de Fibras Empregadas como Reforço 27

$\begin{array}{ll}\text { 2.2.3.1 Fibras Naturais ou Vegetais } & 27\end{array}$

2.2.3.2 Fibras Minerais $\quad 28$

2.2.3.3 Fibras Metálicas $\quad 29$

2.2.3.4 Fibras Poliméricas $\quad 30$

2.2.4 Mecanismos de Interação Solo-Fibra - Estudos Experimentais 31

2.2.5 Alterações nas Propriedades dos Solos pela Inclusão de Fibras $\quad 41$

2.2.5.1 Compactação 41

2.2.5.2 Resistência ao Cisalhamento de Pico 42

2.2.5.3 Resistência ao Cisalhamento pós-pico 45

2.2.5.4 Deformabilidade $\quad 45$

2.2.5.5 Modo de Ruptura 46

2.2.5.6 Variação Volumétrica $\quad 47$

2.2.5.7 Rigidez Inicial $\quad 47$

2.2.5.8 Condutividade Hidráulica e outras Propriedades 48

2.3 Ensaio Triaxial de Extensão 49

3 Programa Experimental 52

3.1 Considerações Iniciais $\quad 52$

3.2 Materiais Utilizados 53

3.2.1 Areia 53

3.2.2 Fibras $\quad 54$

3.2.3 Água $\quad 55$ 
3.3 Equipamento Triaxial Desenvolvido 56

3.3.1 Câmara Triaxial 59

$\begin{array}{ll}\text { 3.3.2 Interface Ar/água } & 61\end{array}$

3.3.3 Sistema de Aquisição de Dados e Controle 62

3.3.4 Sistema de Aplicação de Pressões 63

$\begin{array}{ll}3.3 .5 \text { Instrumentação } & 65\end{array}$

3.4 Método de Ensaio $\quad 66$

3.4.1 Preparação das Amostras para Ensaios Triaxiais 66

$\begin{array}{ll}\text { 3.4.2 Ensaios Triaxiais de Extensão } & 67\end{array}$

4 Apresentação e Discussão dos Resultados 71

4.1 Considerações Iniciais 71

4.2 Definições e Notações Básicas $\quad 71$

4.3 Ensaios de Compressão Triaxial $\quad 72$

4.4 Ensaios de Extensão Triaxial 76

4.4.1 Mecanismo de Deformação 76

4.4.2 Comportamento Tensão Desviadora e Deformação Volumétrica vs Deformação Axial $\quad 78$

4.4.3 Envoltórias e Parâmetros de Resistência ao Cisalhamento 83

4.4.4 Energia de Deformação Absorvida 86

4.4.5 Alongamento das Fibras 88

5 Considerações Finais $\quad 91$

5.1 Conclusões 91

5.2 Sugestões para pesquisas futuras 92

$\begin{array}{ll}\text { Referências Bibliográficas } & 94\end{array}$ 


\section{Lista de Figuras}

Figura 1.1. Ensaios de laboratório a serem realizados ao solo segundo a sua localização ao longo da superfície de ruptura (Fonte: Notas de aula prof. Cezar Bastos. DMC/FURG)

Figura 2.1. Classificação de materiais compósitos (Matthews e Rawlings, 1994)

Figura 2.2. Disposição Fibra/fissura idealizada (Taylor, 1994)

Fifura 2.3. Acréscimo de resistência em função da inclinação da fibra (Gray \& Ohashi, 1983)

Figura 3.1. Curva granulométrica da areia de Osório

Figura 3.2. Aspecto das fibras de polipropileno com $19.3 \mathrm{~mm}$ de comprimento

Figura 3.3. Esquema diagramático do aparelho desenvolvido por Bishop e Wesley

Figura 3.4. Pedestal e top cap do equipamento desenvolvido. (a) estado inicial das peças. (b) alterações desenvolvidas nas peças.

Figura 3.5. (a) Vista geral do equipamento triaxial de maiores dimensões

(b) Câmara triaxial

Figura 3.6. Interface ar/água do equipamento

Figura 3.7. Sistema de aquisição de dados desenvolvido na Imperial College

Figura 3.8. Caixas que regulam as pressões que são aplicadas durante 0 ensaio

Figura 3.9 Bomba de vazão constante de água

Figura 3.10. Mistura da areia com as fibras e a água necessária para atingir $10 \%$ de umidade

Figura 3.11. ( $a$ e b) Moldagem do corpo de prova. (c) Detalhe do equipamento triaxial com a amostra pronta para iniciar o ensaio

Figura 4.1. Parâmetros de resistência da areia submetida a ensaios de compressão obtidos por: (a) CASAGRANDE 2005, (b) presente dissertação Figura 4.2. Parámetros de resistência da mistura submetida a ensaios de compressão obtidos por: (a) CASAGRANDE 2005, (b) presente dissertação 
Figura 4.3. Mecanismo de deformação da amostra da areia submetida a ensaios de extensão

Figura 4.4. Mecanismo de deformação da amostra da mistura areia-fibra submetida a ensaios de extensão

Figura 4.5. Gráfico tensão desviadora vs deformação axial nas tensões efetivas médias normais (a) $50 \mathrm{kPa}$, (b) $100 \mathrm{kPa}$, (c) $200 \mathrm{kPa}$ e (d) $300 \mathrm{kPa}$

Figura 4.6. Curvas tensão desviadora vs deformação axial de todos os ensaios triaxiais drenados de extensão realizados

Figura 4.7. Estado final do corpo de prova da mistura areia-fibra

Figura 4.8. Variação volumétrica vs Deformação axial (a) na areia, (b) na mistura areia - fibra

Figura 4.9. Envoltória e parâmetros de resistência da areia submetida a ensaios triaxiais de (a) compressão (fonte: Casagrande 2005) e (b) extensão

Figura 4.10. Envoltória e parâmetros de resistência da mistura areia-fibra submetida a ensaios triaxiais de (a) compressão (fonte: Casagrande 2005) e (b) extensão

Figura 4.11. Energia de deformação absorvida nos ensaios triaxiais de extensão realizados à areia e à mistura areia - fibra

Figura 4.12. Comprimento final das fibras vs Tensão confinante dos ensaios executados

Figura 4.13. (a) Comprimento inicial das fibras, (b) medida do comprimento final das fibras 


\section{Lista de Tabelas}

Tabela 2.1 - Propriedades físicas e mecânicas das fibras naturais (Braga, 2001)

Tabela 2.2 - Propriedades físicas e mecânicas das fibras minerais (Curcio, 2001)

Tabela 2.3 - Propriedades mecânicas das fibras poliméricas (Mano, 1991;

Hull e Clyne, 1996; Heineck, 2002)

Tabela 3.1 - Indices físicos da areia de Osório

Tabela 3.2 - Resumo das propriedades mecânicas das fibras

Tabela 4.1 - Comparação dos parâmetros de resistência obtidos por CASAGRANDE 2005 e dos resultados obtidos no presente estudo nos ensaios triaxiais drenados de compressão

Tabela 4.2 - Parâmetros de resistência obtidos nos ensaios triaxiais de extensão

Tabela 4.3 - Variação da energia de deformação absorvida com as tensões efetivas médias iniciais 


\section{Lista de Abreviaturas}

ABNT Associação Brasileira de Normas Técnicas

ASTM American Society for Testing and Materials

CAPES Coordenação de Aperfeiçoamento de Pessoal de Nível Superior

CBR California Bearing Ratio

IP Índice de Plasticidade

KEVLAR Fibras de Poliamida

LGMA Laboratório de Geotecnia e Meio Ambiente

LL $\quad$ Limite de Liquidez

LP Limite de Plasticidade

LGMA Laboratório de Geotecnia e Meio Ambiente

LVDT Linearly Variable Differential Transformer

MEF Método dos Elementos Finitos

MVVT Medidor de Variação de Volume Total

NBR Norma Brasileira

PEAD Polietileno de alta Densidade

PET Polietileno Tereftalato 


\section{Lista de Símbolos}

\begin{tabular}{|c|c|}
\hline$\omega_{\text {ótm }}$ & Teor de umidade ótimo de compactação \\
\hline$\gamma_{\mathrm{d} \text { máx }}$ & Peso específico seco aparente máximo \\
\hline$\gamma_{d}$ & Massa específica seca \\
\hline$\gamma_{\mathrm{s}}$ & Densidade real dos grãos \\
\hline$\omega$ & Teor de umidade \\
\hline$\rho$ & Massa específica do solo \\
\hline G & Módulo de cisalhamento \\
\hline $\mathrm{G}_{0}$ & Módulo de cisalhamento inicial ou elástico \\
\hline $\mathrm{G}_{\text {máx }}$ & Módulo de cisalhamento máximo \\
\hline e & Índice de vazios \\
\hline$e_{0}$ & Índice de vazios inicial \\
\hline$E$ & Módulo de deformabilidade ou módulo de Young \\
\hline Edef & Energia de deformação absorvida \\
\hline$v$ & Módulo de Poisson \\
\hline $\mathrm{C}_{\mathrm{u}}$ & Coeficiente de uniformidade \\
\hline Cc & Coeficiente de curvatura \\
\hline $\mathrm{C}_{\mathrm{v}}$ & Coeficiente de adensamento \\
\hline $\mathrm{D}_{10}$ & Diâmetro efetivo \\
\hline $\mathrm{D}_{50}$ & Diâmetro médio \\
\hline$t_{r}$ & Tempo mínimo de ruptura \\
\hline $\mathrm{L}$ & Altura do corpo de prova \\
\hline $1 / d$ & Fator de forma (relação entre o comprimento e o diâmetro) \\
\hline v & Velocidade de cisalhamento \\
\hline ، & Relativo a tensões efetivas \\
\hline$\varepsilon_{\mathrm{a}}$ & Deformação axial \\
\hline$\varepsilon_{\text {rup }}$ & Deformação axial na ruptura \\
\hline$\varepsilon_{\mathrm{v}}$ & Deformação volumétrica \\
\hline$\tau$ & Tensão de cisalhamento \\
\hline$\sigma_{\mathrm{v}}$ & Tensão vertical \\
\hline$\sigma_{\mathrm{h}}$ & Tensão horizontal \\
\hline
\end{tabular}




$\begin{array}{ll}\sigma_{1,} \sigma_{3} & \text { Tensões principais, maior e menor } \\ \sigma_{c} & \text { Tensão de confinamento } \\ \sigma_{d} & \text { Tensão desviadora } \\ u & \text { Poro-pressão } \\ \mathrm{S}_{u} & \text { Resistência não Drenada } \\ \varnothing & \text { Ângulo de atrito } \\ \mathrm{c} & \text { Coesão } \\ \mathrm{p}^{\prime} & \left(\sigma_{1}^{\prime}+2 \sigma_{3}^{\prime}\right) / 3 \text { (Tensão efetiva média normal) } \\ \mathrm{q} & \left(\sigma_{1}^{\prime}-\sigma_{3}^{\prime}\right)(\text { Tensão de Desvio) } \\ \mathrm{M} & \text { Inclinação da envoltória de resistência no espaço p' vs q } \\ \mathrm{h} & \text { Altura final do corpo de prova. } \\ \mathrm{h}_{\mathrm{i}} & \text { Altura inicial do corpo de prova. }\end{array}$

